\section{Medial Lemniscus}

John E. Mendoza

Department of Psychiatry and Neuroscience, Tulane Medical School and SE Louisiana

Veterans Healthcare System, New Orleans, LA, USA

\section{Definition}

A paired somatosensory pathway that originates in the medulla and proceeds rostrally through the central and later the dorsolateral brainstem to the ventral posterior lateral nuclei of the thalamus. The medial lemniscus is formed in the central portion of the medulla by the crossing internal arcuate fibers exiting the cuneate and gracilis nuclei. Thus, when seen in the brainstem, the medial lemniscus represents information from the contralateral side of the body (upper and lower extremities and trunk). Since the nucleus cuneatus and nucleus gracilis derive their input from the posterior (dorsal) columns, the medial lemniscus carries proprioceptive, vibratory, and fine stereognostic (e.g., two-point discrimination) information. By the mid-pontine level the medial lemniscus and the spinothalamic tracts become more or less contiguous. Thus, a single lesion here or higher in the brainstem, in addition to problems in the above cited functions, could also result in loss of pain and temperature perception if the spinothalamic tracts are affected.

\section{Cross-References}

Posterior Columns

- Somatosensory System 